\title{
ULTRARAPID NONSUTURE MATED CUFF TECHNIQUE FOR RENAL TRANSPLANTATION IN RABBITS
}

\author{
JUN WU, M.D., Ph.D., XIAN GE, M.D., Ph.D., \\ AND GREGORY M. FAHY, Ph.D.*
}

An effective and ultrarapid technique for kidney transplantation in the rabbit is introduced. Vascular anastomosis was completed using a novel cuff technique in which mating cuffs were used to join the delicate renal vein. The ureter was reconstructed by spatulated end-to-end anastomosis, with special attention to the rabbit's unique ureteral vascular anatomy. The total vascular anastomosis time was $3.4 \pm 1.3$ min, and there were no episodes of bleeding or thrombosis. The ureter complication rate was $7.3 \%$. Kidneys transplanted after $5 \mathrm{~h}$ of cold storage using the new technique yielded better postoperative creatinine results than similar preserved kidneys transplanted using previously described methods. We suggest this technique for studies of long- and short-term kidney preservation and transplantation in the rabbit, as well as for veterinary transplantation in which donor kidneys must be stored for only a short time before use.

MICROSURGERY 23:369-373
The rabbit is an attractive model for kidney transplantation research, in view of its docility, convenient size, easy maintenance, low cost, and renal anatomical and functional similarity to human kidneys. ${ }^{1-3}$ We were further attracted to the rabbit kidney transplant model due to the overwhelming use of this model in studies involving renal cryopreservation. ${ }^{4-8}$ Most of the publications describing rabbit kidney transplantation techniques utilized microsurgical techniques developed $25-30$ years ago, especially by Green ${ }^{9}$ and Dunn. ${ }^{10}$ In those studies, the warm ischemic time was reported to be between 20-30 min. Since then, few publications have commented on the warm ischemic time. Khirabadi and Fahy ${ }^{11}$ adopted the traditional cuff technique in their rabbit kidney transplantation model, cutting the time for vascular reconstruction to $10-15 \mathrm{~min}$, but this still represents substantial warm ischemia.

The difficulty of rabbit renal vascular anastomosis is mainly due to the delicate nature of the renal vein. We describe a technique that permits ultrarapid $(\sim 3 \mathrm{~min})$ vascular anastomosis, the fastest method so far published, with excellent postoperative patency. The new anastomosis method extends earlier cuff approaches ${ }^{11}$

21st Century Medicine, Inc., Rancho Cucamonga, CA

Grant sponsor: 21st Century Medicine, Inc.

${ }^{*}$ Correspondence to: Gregory M. Fahy, Ph.D., 21st Century Medicine, Inc. 10844 Edison Court, Rancho Cucamonga, CA 91730. E-mail: gfahy@ $21 \mathrm{~cm} . \mathrm{com}$

Received 1 March 2003; Accepted 12 May 2003

Published online in Wiley InterScience (www.interscience.wiley.com). DOI: 10.1002/micr.10145 by adding two mating venous cuffs that can be "plugged" together and secured in a process lasting less than 1 min, without using a loupe or suturing. Another common technical problem with rabbit kidney transplantation is ureter obstruction, ${ }^{12}$ which ranges from $10-30 \%$ postoperatively. We therefore also report on a modified technique for ureter reconstruction that better preserves ureteral blood flow and yields improved patency rates.

These improvements collectively provide a new, controllable, and simplified rabbit renal transplantation model.

\section{MATERIALS AND METHODS}

All procedures involving animals were consistent with the guidelines established by the Animal Care and Use Committee of 21st Century Medicine, Inc., and the principles set forth in the Guide for the Care and Use of Laboratory Animals (Institute of Laboratory Animal Resources, National Research Council, DHHS, publication no. (NIH) 86-23 (1985)) and USDA guidelines.

Male New Zealand White rabbits (Harlan Sprague Dawley, Indianapolis, IN) weighing $2.5-3.1 \mathrm{~kg}$ were housed in separate cages under standard vivarium conditions, and were allowed free access to water and chow. On the day of surgery, rabbits were premedicated with intramuscular ketamine $(30 \mathrm{mg} / \mathrm{kg})$ and xylazine $(5 \mathrm{mg} /$ $\mathrm{kg}$ ) (Henry Schein, Inc., Melville, NY). General anesthesia was maintained by inhalation of $1.5-5 \%$ isoflurane in oxygen at a flow rate of $1.5 \mathrm{~L} / \mathrm{min}$ via a face mask. Rectal temperature was maintained between $36-38.5^{\circ} \mathrm{C}$, using a heating pad and warm saline for irrigating abdominal tissues. Heart rate and saturated oxygen status were 


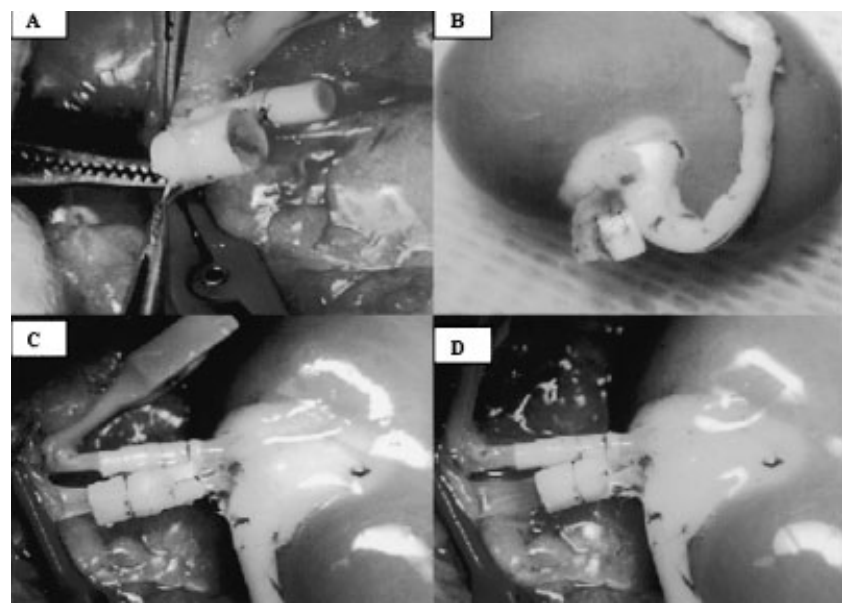

Figure 1. A: Both recipient vessels were passed through and everted over cuffs and secured with circular 6-0 sutures. B: Donor vein was cuffed and secured with two 6-0 prolene sutures while kidney was in cold preservation solution. C: Arterial anastomosis was performed by passing donor kidney's artery over recipient arterial cuff and securing it with a prepositioned circular 6-0 prolene suture. Cuff on donor vein was then initially positioned in recipient venous cuff in an expanded (3.3 $\mathrm{mm} I D)$ segment of the latter, allowing easy insertion and secure orientation of male cuff. D: To create permanent anastomosis, male cuff was then driven fully into female cuff. No further stabilization was required.

monitored by a pulse oximeter (Vet/OX ${ }^{\circledR} 4404$, Heska Corp., Fort Collins, CO), with the probe placed on the tail. Arterial pressure was measured using a 20 -gauge i.v. canula inserted into the central ear artery.

Two separate surgeries were performed for each transplant: a morning surgery for organ procurement, and an afternoon surgery for transplantation. During the morning surgery, the rabbit was hydrated with about 50 $\mathrm{ml}$ of lactated Ringer's solution by slow i.v. drip. During the afternoon surgery, each rabbit received about $150 \mathrm{ml}$ of lactated Ringer's solution in a similar fashion.

\section{Donor Kidney Procurement}

A midventral laparotomy was performed under sterile conditions. After the intestine was covered and protected with warm saline-soaked gauze, the right kidney was exposed. Chlorpromazine ( $2.5 \mathrm{mg}$ dissolved in $2 \mathrm{ml}$ saline) was injected around the renal vessels to avoid vasospasm during dissection, and then the right kidney and its vasculature were gently dissected free from surrounding fat and connective tissue. The ureter was transected approximately $5 \mathrm{~cm}$ from the hilum. A short cannula (18-gauge Vialon i.v. catheter, $2.0 \mathrm{~cm}$ long, Becton Dickinson, Sandy, UT) was inserted into the ureter and ligated in place to permit ready visualization of urine output. Mannitol (2.4 $\mathrm{ml}$ of a $25 \% \mathrm{w} / \mathrm{v}$ solution) and furosemide
$(0.2 \mathrm{ml}$ of $25 \mathrm{mg} / \mathrm{ml})$ were administered intravenously to establish diuresis before ligating the renal artery and then the vein. Immediately after ligation, the renal vein was severed, and the artery was incised and cannulated in situ with a 16-gauge i.v. catheter (inner diameter (ID), 1.16 $\mathrm{mm}$; outer diameter (OD), $1.70 \mathrm{~mm}$; Becton Dickinson). The kidney was flushed at a pressure of $60 \mathrm{mmHg}$ with 50 $\mathrm{ml}$ of $0^{\circ} \mathrm{C}$ storage solution (Eurocollins, UW, or others) containing heparin $(10 \mathrm{U} / \mathrm{ml})$ and chlorpromazine $(50 \mu \mathrm{g} /$ $\mathrm{ml})$. During the initial cold flush, the artery was ligated onto the cannula and cut between the body and the cannula, at which point the kidney was removed and transferred into the selected cold solution at $0^{\circ} \mathrm{C}$. A second flush was then carried out, using another $50 \mathrm{ml}$ of cold solution containing no added drugs. The laparotomy was closed in two layers with 2-0 Vicryl for the abdominal wall and 3-0 Vicryl for the skin. The kidneys were stored in the storage solution $(900 \mathrm{ml})$ at $0^{\circ} \mathrm{C}$ for $5 \mathrm{~h}$. The time for kidney retrieval surgery (from "skin to skin") was 50-60 $\mathrm{min}$. The warm ischemic period during renal procurement was about $1 \mathrm{~min}$.

\section{Kidney Transplantation}

Kidney transplantation was performed approximately $5 \mathrm{~h}$ after nephrectomy. The donor rabbit was reanesthetized and reopened under sterile conditions. A peritoneal flap covering the left kidney and ureter was prepared by cutting along the left renal vessels and the aorta to expose the left ureter and kidney. After injection of chlorpromazine around the renal artery and vein, both vessels were isolated. The kidney was stripped of surrounding connective tissue. The left renal vessels were clamped atraumatically at their origins close to the aorta and vena cava using microvascular clamps, and transected as close as possible to the hilum. The ureter was ligated close to the kidney to avoid damaging the ureteral vein connecting the middle ureter to the vena cava, ${ }^{13}$ and to ensure sufficient ureteral length to preclude traction during the subsequent ureteroureterostomy. The left kidney was then removed and discarded.

The lumens of the left renal artery and vein remaining in the animal were flushed with warm saline. Both vessels were then passed through and everted over cuffs and secured with circular 6-0 sutures (Fig. 1A). The arterial cuff was made of a 14-gauge Surflo i.v. catheter segment (ID, $1.73 \mathrm{~mm}$; OD, $2.17 \mathrm{~mm}$; length, $\sim 5 \mathrm{~mm}$; Terumo Medical Corp., Elkton, MD). The venous cuff was about $5 \mathrm{~mm}$ in length and was made of 10-gauge Angiocath ${ }^{\mathrm{TM}}$ I.V. catheter (ID, $3.0 \mathrm{~mm}$; OD, $3.2 \mathrm{~mm}$; Becton Dickinson), with the ID enlarged by manual distension to $\sim 3.2 \mathrm{~mm}$ along most of its length, and to $\sim 3.3 \mathrm{~mm}$ at the end designated for receiving the kidney. 
The vein of the preserved kidney was cuffed while the kidney remained ex vivo in the $0^{\circ} \mathrm{C}$ storage solution (Fig. 1B). The ex vivo venous cuff was made from 10gauge Angiocath ${ }^{\mathrm{TM}}$ material without expanding its diameter, so that its outer diameter approximated the inner diameter of the in vivo venous cuff. The ex vivo venous cuff was secured with two 6-0 sutures. The two venous cuffs were designed so as to allow the ex vivo (male) cuff and the in vivo (female) cuff to mate.

Shortly before grafting the kidney, $1.6 \mathrm{ml}$ of $25 \%$ mannitol solution were administered intravenously. The preserved kidney was then placed in the peritoneal cavity at the site of the original left kidney. Arterial anastomosis was performed by passing the donor kidney's artery over the recipient arterial cuff and securing it with a prepositioned circular 6-0 prolene suture. Venous anastomosis was then completed by simple insertion of the donor cuffed vein into the recipient cuffed vein, with special attention to vascular orientation on both sides of the anastomosis. The initial cuff-positioning step (Fig. 1C) was facilitated by the expanded (3.3 mm ID) segment of the female cuff, allowing easy insertion and secure orientation of the male cuff. To create a permanent anastomosis, the male cuff was then driven into the female cuff by exerting greater force, applied for example by a spatula pressing on the trailing edge of the advancing male cuff, until the male cuff was fully seated in the female cuff (Fig. 1D). Generally, no further securing sutures were required to maintain the venous anastomosis, but a single suture was sufficient to accomplish stabilization when desired. The vascular clamps were released immediately after fully seating the male cuff in the female cuff.

The ureter was reconstructed over an 8-mm length of 18-gauge Vialon i.v. catheter (Becton Dickinson) placed into the adjoining ureter segments. Special attention was paid to preserving the unique ureteral vein in rabbits that connects the middle ureter to the vena cava. ${ }^{13}$ Eight interrupted sutures of 9-0 nylon were used to complete a tension-free spatulated anastomosis.

At the end of vascular and ureteral anastomosis, the reconstructed ureter and the kidney were covered by the peritoneal flap to avoid postoperative adhesions and to secure their positions, and the flap was sutured in place. The abdominal muscle was closed, as described earlier. The skin was sutured in running fashion with a 3-0 Ethilon suture. Renal transplantation required $80-100$ min from "skin to skin."

\section{Postoperative Maintenance and Follow-Up}

After recovering from anesthesia, rabbits were allowed free access to water and chow. Blood samples $(0.5$ $\mathrm{ml}$ ) were drawn daily via the ear artery for determination of serum levels of blood urean nitrogen (BUN), creatinine, and other chemistries. After approximately 2 weeks of postoperative monitoring, rabbits were again anesthetized and opened, and the grafts were quickly removed and flushed free of blood, using $50 \mathrm{ml}$ of saline containing 5,000 units of heparin at $60-80 \mathrm{mmHg}$. Kidneys were then fixed by perfusion with $50 \mathrm{ml}$ of modified Karnovsky's fixative $(\sim 730 \mathrm{mOsm})$ at the same pressure. Fixed kidneys were sent to Paragon Bioservices (Baltimore, MD) for routine histological processing and periodic acid-Schiff (PAS) staining.

\section{RESULTS}

The results of 113 consecutive renal autografts are summarized. Of these, $109(96.5 \%)$ rabbits survived the anesthesia and transplantation procedure, and $84.4 \%$ of the surviving rabbits were followed for 14-30 days without incident. The combined arterial and venous vascular anastomosis time was $3.4 \pm 1.3$ min (mean \pm $\mathrm{SD})$. There were no episodes of venous or arterial thrombosis or leakage in this series. Incisional hernia, sepsis, wound infection, and hind limb paralysis were not observed in the present series. The only complication noted was ureter blockage, which was evidenced by rising serum creatinine levels in 8 cases out of 109 (7.3\%) on postoperative days 5-10. Most of these incidents recovered spontaneously.

The functional performance of kidneys preserved at $0^{\circ} \mathrm{C}$ in Eurocollins solution for $5 \mathrm{~h}$ and transplanted as described here is compared in Table 1 to historical data obtained using our previous surgical model and to results obtained with sham-operated controls. ${ }^{11}$ The postoperative peak creatinine level resulting from shortterm preservation with the present surgical model was significantly lower than previously reported results $(P<$ 0.01 ), and was in fact even lower than the peak observed in previous sham-operated controls. In addition, serum creatinine returned to normal in 2 days in this model, as compared to 9 days in the previous study.

\section{DISCUSSION}

In this model, rabbits underwent two major surgical interventions in 1 day. The overall survival rate was $96.5 \%$, which exceeds previously reported survival rates. ${ }^{11}$ The only surgical complication was ureter blockage, which occurred in $7.3 \%$ of the procedures, compared to an incidence of $10-30 \%$ in the literature. ${ }^{10,12,14,15}$ The mean peak creatinine level for kidneys stored in Eurocollins solution for $5 \mathrm{~h}$ was significantly lower than previous results, and even better than results 
Table 1. Postoperative Serum Creatinine Levels*

\begin{tabular}{|c|c|c|c|}
\hline & 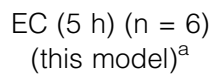 & $\begin{array}{l}\mathrm{EC}(5 \mathrm{~h})(\mathrm{n}=7) \\
\text { (historical data) }^{\mathrm{b}}\end{array}$ & $\begin{array}{l}\text { Sham operation }(\mathrm{n}=6) \\
{\text { (historical data })^{\mathrm{b}}}^{\text {(hicto }}\end{array}$ \\
\hline Peak serum creatinine (mg/dl) & $2.02 \pm 0.72^{\mathrm{C}}$ & $6.81 \pm 3.13$ & $3.76 \pm 3.43$ \\
\hline Days to peak & $1.0 \pm 0.0$ & $2.7 \pm 1.1$ & $1.5 \pm 0.6$ \\
\hline Days to new baseline ${ }^{d}$ & $2.2 \pm 0.4^{\mathrm{C}}$ & $9.3 \pm 1.1$ & $5.8 \pm 1.8$ \\
\hline Preoperative creatinine (mg/dl) & $0.68 \pm 0.12$ & $1.41 \pm 0.10$ & $1.15 \pm 0.07$ \\
\hline Final creatinine $(\mathrm{mg} / \mathrm{dl})$ & $1.20 \pm 0.22$ & $1.84 \pm 0.15$ & $1.58 \pm 0.13$ \\
\hline Final minus initial creatinine & 0.52 & 0.43 & 0.43 \\
\hline
\end{tabular}

${ }^{\star}$ Mean $\pm S D(m g / d l)$

${ }^{a}$ Eurocollins solution.

${ }^{b}$ Data from Khirabadi and Fahy. ${ }^{11}$

'Significantly different from historical eurocollins (EC) data, $P<0.01$ by Student's $t$-test.

${ }^{d}$ Days to return creatinine to values equal to final values achieved for each specific rabbit.

for sham-operated kidneys in which the kidney was subjected to $1 \mathrm{~min}$ of warm ischemia and ureter transection and reanastomosis, but not to any preservation. ${ }^{11}$

\section{Renal Vascular Anastomosis}

Warm ischemia during renal transplantation probably contributes to the overall damage observed after renal cold storage and should therefore be minimized. In this study, the warm ischemic time attributable to nephrectomy was about $1 \mathrm{~min}$, and vascular anastomosis contributed approximately another $3 \mathrm{~min}$ on average to the total warm ischemic time. To our knowledge, this is the fastest vascular reconstruction so far reported in renal transplantation, with normal anastomosis times ranging from about $9-25 \mathrm{~min}$ in rabbit models $^{3,9-11,14,15}$ to $5-30 \mathrm{~min}$ in the rat. ${ }^{16-19}$

The method of vascular anastomosis described here is a further development of our previously described cuff technique, ${ }^{11}$ but using two mated cuffs for renal vein anastomosis. The nonsuture external cuff method was first described in 1900 by Payr. ${ }^{20}$ Modifications of the technique were extensively and successfully used in experimental organ transplantation research involving rabbits and rats. $9,10,20,21$ Theoretically, the cuff technique is faster than end-to-end suturing and avoids problems such as intimal damage, imperfect intima-tointima contact, complications from the presence of intraluminal suture, and incomplete sealing of the anastomosis. One past problem with realizing these theoretical advantages in the rabbit has been the delicate nature of the rabbit renal vein, which has required at least $10 \mathrm{~min}$ to complete cuffing and anastomosis, with a patency rate of $83-99 \% .^{9-11} \mathrm{We}$ overcame this time limitation by using two mating venous cuffs and by preparing all cuffs while the kidney was still in the cold preservation solution. During the actual transplantation procedure, the two mating cuffs were simply "plugged" together in a process which could be completed in less than $1 \mathrm{~min}$. The renal artery, on the other hand, is easy to pass over the arterial cuff and secure, and thus does not need the mating cuff technique for fast anastomosis.

Overall, the current method provides a simple, rapid, and reliable means of vascular anastomosis. The endothelium is continuous across both anastomosis sites, and the presence of the cuff externally may help to prevent posttransplant stenosis. We observed no episodes of vascular thrombosis despite the absence of anticoagulant usage in the described procedures.

\section{Ureter Anastomosis}

A very common technical problem of renal transplantation in the rabbit is the maintenance of adequate ureteral patency. A wide variety of techniques has been reported. Most techniques involve either end-to-end uretostomy or uretero-cystostomy. No matter which technique is used, the ureter complication rate varies from $10-30 \% .^{10,12,14,15}$ Although edema may contribute to ureteral stenosis, ischemia of the suture line and subsequent stricture formation are the main causes of ureter obstruction. ${ }^{22}$ On this basis, we adopted a spatulated end-to-end reconstruction technique, which generates a more distributed anastomosis line that is less susceptible to occlusive stricture. An internal stent facilitated the approximation of the two sides of the ureter and precluded accidental suturing together of the dorsal and ventral ureteral walls. This small stent can be passed into the bladder and then voided without incident most of the time.

We also felt that attention to the anatomy of the rabbit ureter's vasculature could be important for minimizing ureteral ischemia. The rabbit ureter is supplied predominantly by a branch of the renal artery proximally and by a branch of the vesicular artery distally. ${ }^{13}$ The middle segment of the rabbit ureter has no reported extrinsic blood supply, and is drained by a 
single vein emptying into the vena cava. ${ }^{13}$ However, we observed that this "emptying vein" is accompanied by a tiny artery originating from either the aorta or the vesicular artery (unpublished observations). A single group of longitudinal arteries and veins runs the full length of the ureter within the adventitia. Branches of these longitudinal vessels pass tangentially through the muscularis to supply a vascular complex within the lamina propria. ${ }^{13}$ Therefore, in addition to mimimizing any manipulation of the tissue at the hilum, the blood vessels supplying the middle segment of the recipient ureter have to be preserved. In the present series of rabbit renal autografts, in which attention to these considerations was given, only $7.3 \%$ of the grafts showed some degree of ureteral obstruction on postoperative days $5-10$, and most of these complications resolved without active intervention.

\section{CONCLUSIONS}

We suggest this ultrarapid nonsuture mated cuff technique for studies of long- and short-term kidney preservation and transplantation in the rabbit, as well as for veterinary transplantation in which donor kidneys must be stored for only a short time before use.

\section{ACKNOWLEDGMENTS}

We acknowledge the excellent surgical assistance provided by Alice Chang and the helpful technical support of Perlie Tam and John Phan. We thank Richard Infante for critical environmental support in all phases of this work.

\section{REFERENCES}

1. Andrews PM, Khirabadi BS, Bengs BC. Using tandem scanning confocal microscopy to predict the status of donor kidneys. Nephron 2002;91:148-155.

2. Horuk R, Shurey S, Ng HP, May K, Bauman JG, Islam I, Ghannam A, Buckman B, Wei GP, Xu W, Liang M, Rosser M, Dunning L, Hesselgesser J, Snider RM, Morrissey MM, Perez HD, Green C. CCR1-specific non-peptide antagonist: efficacy in a rabbit allograft rejection model. Immunol Lett 2001;76:193-201.
3. Sammut IA, Burton K, Balogun E, Sarathchandra P, Brooks KJ, Bates TE, Green CJ. Time-dependent impairment of mitochondrial function after storage and transplantation of rabbit kidneys. Transplantation 2000;69:1265-1275.

4. Kheirabadi BS, Fahy GM. Permanent life support by kidneys perfused with a vitrifiable (7.5 molar) cryoprotectant solution. Transplantation 2000;70:51-57.

5. Jacobsen IA, Pegg DE, Wusteman MC, Robinson SM. Transplantation of rabbit kidneys perfused with glycerol solutions at 10 degrees C. Cryobiology 1978;15:18-26.

6. Hunt CJ. Studies on cellular structure and ice location in frozen organs and tissues: the use of freeze-substitution and related techniques. Cryobiology 1984;21:385-402.

7. Fahy GM, Ali SE. Cryopreservation of the mammalian kidney. II. Demonstration of immediate ex vivo function after introduction and removal of 7.5 M cryoprotectant. Cryobiology 1997;35:114131 .

8. Arnaud FG, Khirabadi BS, Fahy GM. Normothermic blood perfusion of isolated rabbit kidneys. III. In vitro physiology of kidneys after perfusion with Euro-Collins solution or $7.5 \mathrm{M}$ cryoprotectant (VS4). Transplant Int 2002;15:278-289.

9. Green CJ. Rabbit renal autografts as an organ preservation model. J Lab Anim 1973;7:1-11.

10. Dunn DC. Orthotopic renal transplantation in the rabbit. Transplantation 1976;22:427.

11. Khirabadi BS, Fahy GM. Cryopreservation of the mammalian kidney. I. Transplantation of rabbit kidneys perfused with EC and RPS-2 at $2-4^{\circ} \mathrm{C}$. Cryobiology 1994;31:10-25.

12. Nørby J, Jacobsen IA, Pegg DE, Starklint H, Chemnitz J, Diaper MP. Preservation of rabbit kidneys using a solution containing hydrolyzed starch. Transplantation 1991;52:799-804.

13. Douglas G, Hossler FE. Vascular anatomy of the rabbit ureter. Anat Rec 1995;242:47-56.

14. Jacobsen IA. Renal transplantation in the rabbit: A model for preservation studies. Lab Anim 1978;12:63-70.

15. Francis DMA, Millar RJ, Dumble LJ, Clunie GJA. Model of orthotopic renal transplantation in the rabbit. Aust N Z J Surg 1990;60:45-49.

16. Fisher B, Lee S. Microvascular surgical techniques in research, with special reference to renal transplantation in the rat. Surgery 1965;58:904-914.

17. Lee $S$. An improved technique of renal transplantation in the rat. Surgery 1967;61:771-773.

18. Engelbrecht G, Khan D, Duminy F, Hickman R. New rapid technique for renal transplantation in the rat. Microsurgery 1992;13:340-344.

19. Lopez-Neblina F, Toledo-Pereyra LH, Suzuki S. Ultrarapid orthotopic technique for renal transplantation in the rat. Microsurgery 1994;15:274-278.

20. Payr E. Beiträge zur Technik der Blutgefäss- und Nervennaht nebst Mittheilungen über die Verwendung eines resorbirbaren Metalles in der Chirurgie. Arch Klin Chir 1900;62:67.

21. Kamada N. A description of cuff techniques for renal transplantation in the rat. Use in studies of tolerance induction during combined liver grafting. Transplantation 1985;39:93-95.

22. Png JC, Chapple CR. Principles of ureteric reconstruction. Curr Opin Urol 2000;10:207-212. 